\title{
Cerebral metabolic impairment in patients with obstructive sleep apnoea: an independent association of obstructive sleep apnoea with white matter change
}

\author{
M Kamba, Y Inoue, S Higami, Y Suto, T Ogawa, W Chen
}

\begin{abstract}
Objectives-To determine the relation between severity of obstructive sleep apnoea (OSA) and degree of cerebral metabolic impairment.

Methods-Fifty five patients with habitual snoring and excessive daytime sleepiness underwent standard overnight polysomnography and magnetic resonance spectroscopy separately. Proton MR spectra were measured with two dimensional chemical shift imaging (repetition time; $1500 \mathrm{~ms}$, echo time; $135 \mathrm{~ms})$. Severity of cerebral metabolic impairment was assessed by the $\mathrm{N}$-acetylaspartate (NAA)/choline ratios for the cerebral cortex and white matter. Severity of OSA was assessed by the apnoea-hypopnoea index (AHI) and the minimum value of peripheral oxyhaemoglobin saturation. All patients were evaluated for the presence or absence of comobidities including hypertension, cardiac disease, diabetes mellitus, and hyperlipidaemia. Univariate analysis of variance (ANOVA) and mulitple linear regression analysis were used for statistical analyses. Results-Univariate ANOVA disclosed significant effects of AHI, age, and the presence or absence of hypertension on the NAA/choline ratio for cerebral white matter $(p=0.011, p=0.028, p=0.0496$, respectively). The AHI had a significant negative association with the NAA/choline ratio for cerebral white matter, independent of age and the presence or absence of cardiac disease, in the final multivariate regression model (standardised partial regression coefficient $=-0.417, p<0.001$ ). No significant relation was found between severity of OSA and the NAA/choline ratio for the cerebral cortex. Age alone had a significant effect on the NAA/choline ratio for the cerebral cortex on univariate ANOVA $(p<0.001)$ and a significant negative association with the NAA/choline ratio for the cerebral cortex in the regression model $(r=-0.552, p<0.001)$.

Conclusions-A significant relation exists between AHI and the degree of metabolic impairment in cerebral white matter in patients with OSA.

(F Neurol Neurosurg Psychiatry 2001;71:334-339)
\end{abstract}

Keywords: magnetic resonance spectroscopy; sleep apnoea syndromes; white matter disease
Repeated sleep apnoeic episodes may lead to CNS impairment in patients with obstructive sleep apnoea (OSA). Excessive daytime sleepiness and cognitive and emotional deficits are common daytime symptoms of OSA. ${ }^{1-6} \mathrm{~A}$ multiple sleep latency test and maintenance of wakefulness test have been used to quantify daytime sleepiness. ${ }^{78}$ Neuropsychological and electrophysiological tests have been used to quantify cognitive dysfunction. ${ }^{1-69-11}$ Hypoxic brain damage and fragmentation of sleep are generally thought to be causes of these deficits. $^{2-689}$

Magnetic resonance spectroscopy (MRS) enables non-invasive evaluation of focal metabolic changes in various conditions affecting the CNS. We previously reported cerebral metabolic changes in patients with OSA. ${ }^{12}$ The $\mathrm{N}$-acetylaspartate (NAA)/choline ratio for cerebral white matter was significantly lower in patients with moderate to severe OSA than in patients with mild OSA and healthy subjects, indicating axonal injury or gliosis in those with the severe form. ${ }^{13-15}$ Our results of MRS examination during sleep disclosed lactate production, indicating that hypoxia is causing anaerobic glycolysis in the brain of patients with moderate to severe OSA. ${ }^{16}$ Our previous results suggested that a relation exists between severity of OSA and the degree of cerebral metabolic change. Patients with OSA often have several comorbidities including hypertension and other cardiovascular diseases, diabetes mellitus, and hyperlipidaemia, which are generally thought to be cerebrovascular risk factors. ${ }^{17-19}$ These comorbidities may promote cerebral ischaemia, and play a part in the occurrence of cerebral metabolic change. Aging is also thought to be related to cerebral metabolic change. ${ }^{20}$ The aims of this study were to investigate which of these factors is significantly related to cerebral metabolic change and to determine the usefulness of MRS as an objective method for evaluation of CNS impairment in patients with OSA.

\section{Methods}

PATIENTS

All patients were recruited at the Sleep Disorder Clinic of Tottori University Hospital. Medical records of potential participants were collected on enrollment. Those with a history of CNS diseases or medical conditions contraindicating MRS such as the presence of a cardiac pacemaker were excluded from the study. Patients who had undergone tracheostomy were also excluded. Sixty three patients 
(55 men and eight women; age 22-75 years, mean 49.13 (SD 13.89) years) with habitual snoring and excessive daytime sleepiness underwent diagnostic polysomnography and MRS in our hospital. All patients were clinically evaluated for the presence or absence of comorbidities including hypertension, cardiac disease, diabetes mellitus, and hyperlipidaemia. Antihypertensive treatment at the time of enrollment or systolic blood pressure greater than $160 \mathrm{~mm} \mathrm{Hg}$ or diastolic blood pressure greater than $95 \mathrm{~mm} \mathrm{Hg}$ on two independent visits was considered evidence of hypertension. A history of documented ischaemic heart disease or abnormal ECG exhibiting ischaemic changes, left ventricular hypertrophy, or arrhythmia was considered evidence of cardiac disease. Antidiabetic treatment at the time of enrollment or fasting blood glucose concentration of $140 \mathrm{mg} / \mathrm{dl}$ or greater was considered evidence of diabetes mellitus. Treatment for hyperlipidaemia at the time of enrollment or total cholesterol concentration of $240 \mathrm{mg} / \mathrm{dl}$ or more was considered evidence of hyperlipidaemia. The body mass index (weight $(\mathrm{kg}) /$ height $^{2}$ $(\mathrm{m})$ ) of each patient was determined at the time of polysomnographic examination. None of the patients had congenital metabolic abnormalities affecting the CNS. After complete description of the study to the patients, written informed consent was obtained in accordance with the Declaration of Helsinki.

\section{DIAGNOSTIC POLYSOMNOGRAPHY}

Standard overnight polysomnography was performed for each patient within 3 months before the MRS study. Sleep state was monitored by EEG, electro-oculography, and submental EMG. Air flow in the nasal and oral cavities was monitored with thermistors. Thoracoabdominal respiratory motion was monitored with strain gauges. Arterial oxygenation was monitored with a Biox II finger pulse oximeter (Ohmeda, Louisville, Colorado, USA). The polysomnographic records were manually assessed using conventional criteria. ${ }^{21}{ }^{22}$ Severity of sleep apnoea was assessed by the apnoeahypopnoea index (AHI) and the minimum value of peripheral oxyhaemoglobin saturation. Hypopnoea was defined as a reduction of breathing during sleep with a decrease in oxyhaemoglobin saturation equal to or more than $3 \%$ from baseline or arousal. ${ }^{23}$

MRS

Magnetic resonance imaging and MRS were performed with a Magnetom Vision with a standard circulatory polarised head coil operating at $1.5 \mathrm{~T}$ (Siemens, Erlangen, Germany). T1 weighted spin echo images were obtained in transverse, coronal, and sagittal directions. T2 weighted turbo spin echo images were obtained in a transverse direction. These MR images were used for anatomical localisation and screening for CNS abnormalities. Patients with abnormal MRI findings including brain infarction, haemorrhage, and white matter hyperintensity lesions were excluded from subsequent MRS examination, as metabolic changes due to OSA are indistinguishable from those caused by such
Table 1 Patient characteristics

\begin{tabular}{ll}
\hline No of patients & 55 \\
Age (y) & $46.45(12.64)$ \\
Men/women & $49 / 6$ \\
Body mass index $\left(\mathrm{kg} / \mathrm{m}^{2}\right)$ & $28.76(5.84)$ \\
Hypertension, yes & $16(29 \%)$ \\
Cardiac disease, yes & $6(11 \%)$ \\
Diabetes mellitus, yes & $7(13 \%)$ \\
Hyperlipidaemia, yes & $8(15 \%)$ \\
Apnoea-hypopnoea index $(/ \mathrm{h})$ & $43.78(30.00)$ \\
SpO ${ }_{2 \text { min }}(\%)$ & $73.40(16.36)$ \\
$N$-acetylaspartate/choline ratio: & \\
$\quad$ Cerebral cortex & $2.18(0.36)$ \\
$\quad$ Cerebral white matter & $1.85(0.21)$ \\
\end{tabular}

Values are means (SD) unless indicated otherwise.

$\mathrm{SpO}_{2 \text { min }}=$ Minimum value of peripheral oxyhaemoglobin saturation.

CNS abnormalities. Patients with only periventricular rim, periventricular cap, or punctate lesions were included among those undergoing subsequent MRS examination. ${ }^{24}$

The MRS examination and postprocessing were performed as described in detail previously. ${ }^{12}$ Two dimensional chemical shift imaging studies $^{25}$ were performed using a $10 \mathrm{~mm}$ slice thickness with $16 \times 16$ phase encoding steps over a field of view of $160 \mathrm{~mm}$ in a transverse plane at the level of the bodies of the lateral ventricles. A volume of interest between $80 \times 80$ and $100 \times 100$ $\mathrm{mm}$ was selected within the field of view. We used a repetition time of $1500 \mathrm{~ms}$ and an echo time of $135 \mathrm{~ms}$ with two signal acquisitions for each phase step, resulting in a measurement time of 12 minutes 55 seconds. To achieve good static magnetic field homogeneity, we used multi-angle projection shim $^{26}$ combined with localised manual shimming of the volume of interest, resulting in a water resonance line width of $5-8 \mathrm{~Hz}$ (full width at half maximum). Magnetic resonance examinations were performed with patients awake.

After acquisition, spectral data were obtained using Luise software (Siemens). Peak areas for NAA $(2.0 \mathrm{ppm})$, creatine $(3.0 \mathrm{ppm})$, and choline $(3.2 \mathrm{ppm})$ were calculated by fitting the spectrum to a sum of gaussian curves. The NAA/choline ratio was calculated from the peak areas. Results from voxels of roughly the anterior half of the volume of interest were discarded to avoid magnetic susceptibility effects. Results from voxels in columns on both ends of the volume of interest were discarded to avoid contamination of fat signal from the skull and scalp. The voxels mainly containing the medial aspects of the occipital and parietal lobes and cingulate gyrus (7 to 10 voxels) and those containing the posterior half of the periventricular white matter ( 8 to 12 voxels) were selected by visual inspection. The mean NAA/choline ratios for the cerebral cortex and those for the periventricular white matter were calculated separately from the selected voxels.

\section{STATISTICS}

Univariate analysis of variance (ANOVA) was used to determine main effects of candidate predictors including age, body mass index, AHI, the minimum value of peripheral oxyhaemoglobin saturation, the presence or absence of hypertension, cardiac disease, diabetes mellitus, and hyperlipidaemia on the NAA/choline 
A

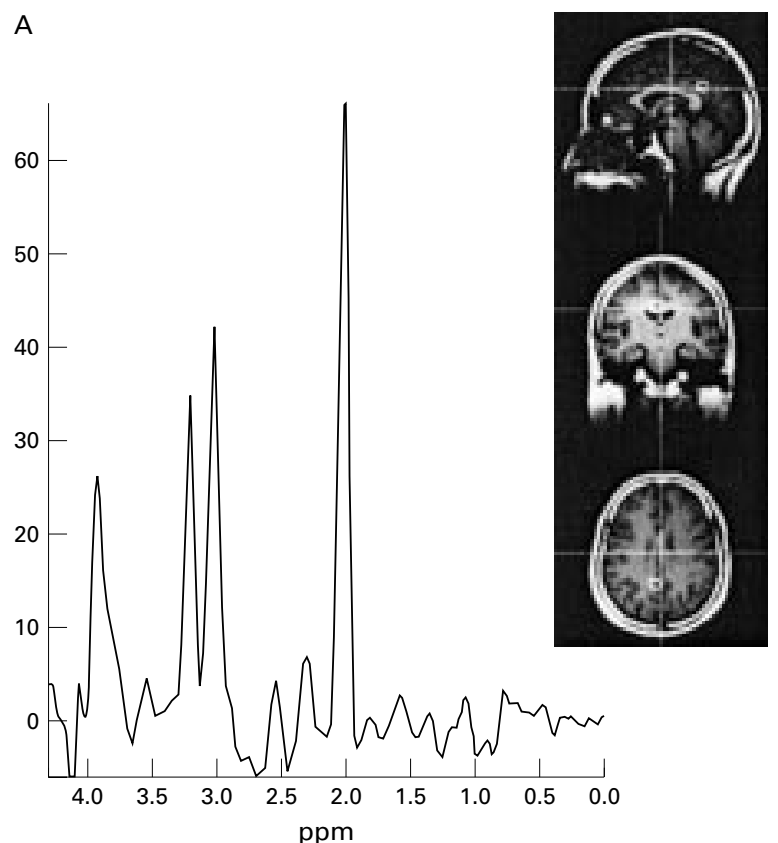

B

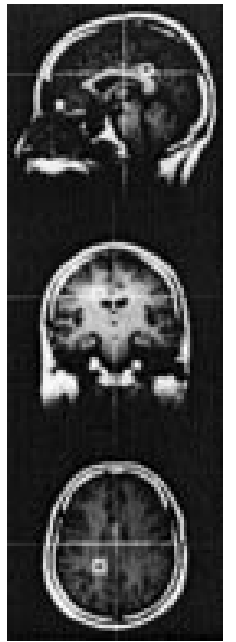

Figure 1 Representative spectra from $(A)$ the cerebral cortex and (B) white matter of a patient with obstructive sleep apnoea. The chemical shift axis is displayed in ppm. The vertical axis is displayed in arbitrary units. Resonances of protons of $N$-acetylaspartate (2.0 ppm), creatine (3.0 ppm), and choline (3.2 ppm) are resolved.

ratios for the cerebral cortex and white matter and interactions between the candidate predictors. The level of significance was set at $p<0.05$. Variables with a main effect of $p<0.20$ in the univariate ANOVA were included in the multiple linear regression analyses for predicting the NAA/choline ratios for the cerebral cortex and white matter. The stepwise method based on $\mathrm{F}$ statistics was used for selection of variables for the final linear regression model. Entry and removal criteria were set at $p \leqslant 0.05$ and $p \geqslant 0.10$, respectively. SPSS for Windows (SPSS Japan, Tokyo, Japan) was used for statistical analyses.

\section{Results}

Eight patients (six men and two women; age range 59-74 years) with abnormal MRI findings were excluded from the MRS study. Thus, results for 55 patients (49 men and six women, age range 22-75 years; mean 46.45 (SD 12.64) years) were analyzed. The characteristics of the patients analyzed are summarised in table 1.

Representative spectra from the cerebral cortex and white matter are shown in figure 1 .

Table 2 Univariate analyses of variance of candidate predictors for the NAA/choline ratios for the cerebral cortex and white matter

\begin{tabular}{|c|c|c|c|c|c|}
\hline & \multirow[b]{2}{*}{$d f$} & \multicolumn{2}{|c|}{$N A A /$ choline $_{\text {cerebral contex }}$} & \multicolumn{2}{|c|}{$N A A /$ choline $_{\text {cerebral white matter }}$} \\
\hline & & F value & $p$ Value & F value & $p$ Value \\
\hline Age & 1,38 & 14.597 & $<0.001$ & 5.220 & 0.028 \\
\hline $\operatorname{Sex}^{\star}$ & 1,38 & 1.006 & 0.322 & 0.216 & 0.644 \\
\hline Body mass index & 1,38 & 0.656 & 0.423 & 1.096 & 0.302 \\
\hline Hypertension ${ }^{\star}$ & 1,38 & 1.829 & 0.184 & 4.111 & 0.0496 \\
\hline Cardiac disease & 1,38 & 0.056 & 0.814 & 1.887 & 0.178 \\
\hline Diabetes mellitus & 1,38 & 0.373 & 0.545 & 0.815 & 0.372 \\
\hline Hyperlipidaemia & 1,38 & 0.079 & 0.780 & 0.758 & 0.389 \\
\hline Apnoea-hypopnoea index & 1,38 & 0.001 & 0.980 & 7.195 & 0.011 \\
\hline $\mathrm{SpO}_{2 \min }$ & 1,38 & 0.093 & 0.762 & 2.149 & 0.151 \\
\hline
\end{tabular}

${ }^{\star}$ The effect of sex $\times$ hypertension interaction on the NAA/choline ratio for the cerebral white matter was significant $(F(1,38)=8.860, \mathrm{p}=0.005)$

$\mathrm{df}=$ Degrees of freedom; $\mathrm{SpO}_{2}$ min $=$ minimum value of peripheral oxyhaemoglobin saturation.
Resonances of protons of NAA, creatine, and choline are resolved.

There was a significant main effect of age on the NAA/choline ratio for the cerebral cortex in the univariate ANOVA (table 2). No other significant main effect or interaction was found for the NAA/choline ratio for the cerebral cortex. Age and the presence or absence of hypertension were included in the multiple linear regression analysis. Age alone was selected for the final linear regression model predicting the NAA/choline ratio for the cerebral cortex. The regression equation was as follows:

NAA/choline ${ }_{\text {cortex }}$ $=-0.0158 \times$ age $($ years $)+2.92$,

$R=0.552, \quad R^{2}=0.305$, adjusted $R^{2}=0.292$, $\mathrm{p}<0.001$ (fig 2).

The main effects of age, AHI, and the presence or absence of hypertension and sex $\times$ hypertension interaction on the NAA/ choline ratio for the cerebral white matter reached significance (table 2). These three variables, the minimum value of peripheral oxyhaemoglobin saturation, and the presence or absence of cardiac disease were included in the multiple linear regression analysis. The AHI, age, and the presence or absence of cardiac disease were selected for the final linear regression model for predicting the NAA/ choline ratio for cerebral white matter. The regression equation was as follows:

NAA/Choline

$=-0.00525 \times$ age (years) $-0.00277 \times \mathrm{AHI}$

(/hour) $-0.194 \times$ cardiac disease +2.23

$R=0.573, \quad R^{2}=0.328$, adjusted $R^{2}=0.289$, $\mathrm{p}<0.001$;

where the presence or absence of cardiac disease was coded 1 or 0 , respectively (fig 3 ). 


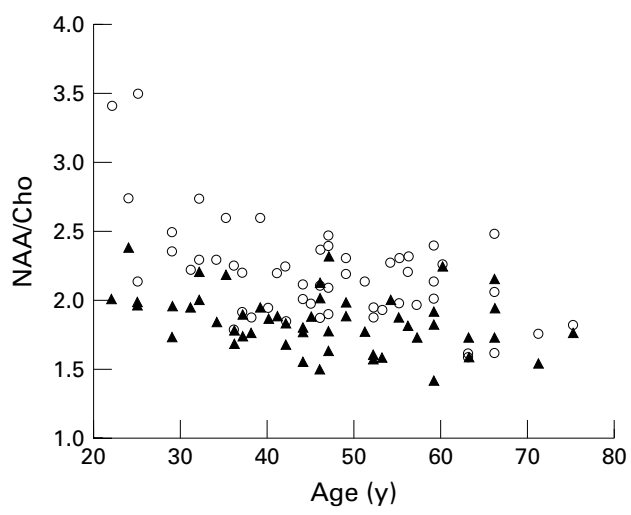

Figure 2 Relations between age and the NAA/choline ratios for the cerebral cortex (open circles) and white matter (closed triangles).

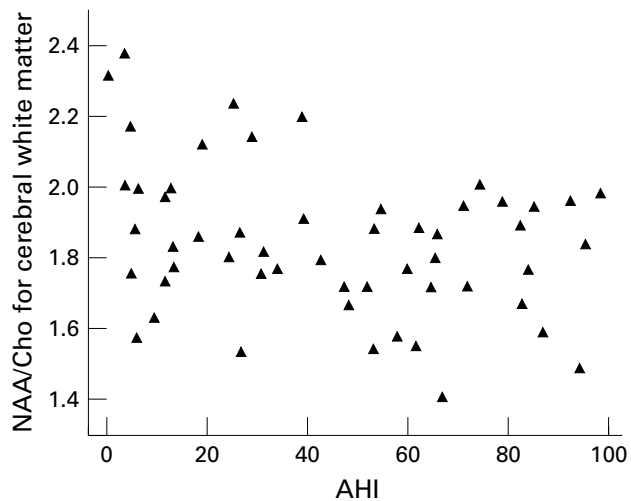

Figiure 3 Relation between the apnoea-hypopnoea index (AHI) and the NAA/choline ratios for cerebral white matter.

Standardised partial regression coefficients for age, AHI, and the presence or absence of cardiac disease were $-0.319(\mathrm{p}=0.011),-0.417$ $(\mathrm{p}<0.001)$, and $-0.300 \quad(\mathrm{p}=0.014)$, respectively. The relation between age and the NAA/ choline ratio for cerebral white matter is shown in figure 2.

\section{Discussion}

We used the NAA/choline ratio as a metabolic indicator of cerebral impairment in this study. It is generally considered that NAA is solely of neuronal and axonal origin. ${ }^{27}{ }^{28}$ Choline resonance is thought to originate mainly from phosphorylated cholines. ${ }^{28} \mathrm{~A}$ decrease in NAA resonance is associated with neuronal loss, or axonal injury, or both. ${ }^{13-15}$ Changes in membrane metabolism and glial cell reaction may increase choline resonance. ${ }^{13-15}$ The NAA/ choline ratio has been used as a sensitive indicator of cerebral metabolic impairment including neuronal loss, axonal injury, and gliosis. ${ }^{13-15}$

Age alone had a significant association with the NAA/choline ratio for the cerebral cortex on the univariate ANOVA and linear regression analysis. The NAA/choline ratio for the cerebral cortex decreased with increasing age. No significant relation was found between severity of OSA and the NAA/choline ratio for the cerebral cortex. Age, AHI, and the presence or absence of hypertension were significantly correlated with the NAA/choline ratio for periventricular white matter on univariate ANOVA.
However, AHI, age, and the presence or absence of cardiac disease were selected for the final linear regression model. The NAA/ choline ratio for periventricular white matter decreased with increasing age and AHI and with the presence of cardiac disease. These findings indicate that an independent association exists between AHI and metabolic impairment in the periventricular white matter, which seems normal on the MRI of patients with OSA.

Arterial oxyhaemoglobin saturation may decrease during apnoea. A known mechanism of cerebral circulatory regulation increases cerebral blood flow in response to hypercapnia. Our results of MRS examination during sleep disclosed lactate production, indicating hypoxia causing anaerobic glycolysis, in the cerebral deep white matter of patients with moderate to severe OSA. ${ }^{16}$ This finding indicates that haemodynamic adjustment in the cerebral deep white matter may be not sufficient to compensate for a decrease in arterial oxyhaemoglobin saturation, and supports the hypothesis that cerebral hypoxia due to repeated sleep apnoeic episodes is responsible for the metabolic impairment seen in the cerebral deep white matter. However, the minimum value of oxyhaemoglobin saturation was not significantly related to the decrease in the NAA/choline ratio or the production of lactate. ${ }^{16}$ Results of several studies using transcranial Doppler sonography have indicated changes in cerebral circulatory adjustment in patients with OSA, including reduced cerebral blood flow velocity in response to obstructive sleep apnoeic episodes and altered cerebrovascular reactivity to hypercapnia. ${ }^{29-32}$ These findings suggest that cerebral metabolic impairment due to OSA cannot be explained by a simple linear relation with arterial oxyhaemoglobin saturation.

The susceptibility of the cerebral deep white matter to OSA may be explainable in part by features of vessels in this region. Cerebral deep white matter is supplied via long small arteries and arterioles. ${ }^{33}$ These vessels in white matter are subject to sclerotic changes, particularly in aged people. ${ }^{34}$ In the cerebral deep white matter, the cerebrovascular reserve may not be sufficient to compensate for decreases in arterial oxyhaemoglobin saturation during repeated sleep apnoeic episodes.

Patients with OSA often have several comorbidities related to the cardiovascular system and metabolism including hypertension, cardiac diseases, and diabetes mellitus, which are thought to be cerebrovascular risk factors. ${ }^{17-19}$ Hypertension and diabetes mellitus are generally thought to accelerate arteriolosclerosis. ${ }^{34} 35$ Cardiovascular and metabolic complications of OSA probably promote cerebral ischaemia, and play a part in the occurrence of metabolic impairment in the cerebral deep white matter. Our findings suggest that cerebrovascular risk factors may play a part in the occurrence of cerebral metabolic impairment.

Our finding of no significant relation between severity of OSA and the NAA/choline ratio for the cerebral cortex suggests that the 
haemodynamic adjustment in the cerebral cortex may be sufficient to compensate for the decrease in arterial oxyhaemoglobin saturation during sleep apnoeic episodes. This finding may not hold true of other brain regions, such as the watershed areas and hippocampus. It is possible that an association existed between severity of OSA and the NAA/choline ratio for the cerebral cortex that was too weak to be detected in our study. The relatively large SD of the NAA/choline ratio for the cerebral cortex may to some extent be responsible for the lack of a relation between the severity of OSA and the NAA/choline ratio for this region. Incomplete separation of the cerebal cortex from white matter and CSF due to the low spatial resolution of our MRS examination may have resulted in considerable error in the determination of the NAA/choline ratio for the cerebral cortex. Further investigations are needed to determine the relation between severity of OSA and metabolic impairment in the cerebral cortex.

Many studies have shown cognitive and neuropsychological dysfunction in patients with OSA. ${ }^{1-6} 9-11$ Treatment with continuous positive airway pressure may improve these deficits, but not normalise them completely. ${ }^{4} 910$ Hypoxaemia during sleep and fragmentation of sleep are generally thought to play a part in causing these deficits. ${ }^{2-69}$ On the other hand, cerebral white matter lesions on MRI, which are generally thought to be related to chronic cerebral ischaemia, are often found in the aged, particularly those with hypertension or other cardiovascular or metabolic diseases. ${ }^{35}$ Many studies have found a relation between cerebral white matter lesions and cognitive and neuropsychological dysfunction. ${ }^{3637}$ Our MRS finding of metabolic impairment in the cerebral deep white matter may be related to cognitive and neuropsychological dysfunction in patients with OSA. Our results, however, failed to demonstrate an association of OSA with cortical degeneration. A comparative study of MRS and results of neuropsychological examinations is needed to determine the relation of cerebral metabolic impairment to cognitive and neuropsychological dysfunction in patients with OSA.

Because our study was cross sectional, the causal relation between OSA and the metabolic impairment in cerebral white matter cannot be determined from our results. Further investigations by longitudinal and experimental studies are needed to determine this.

In summary, our MRS findings indicate that a significant relation exists between AHI and the degree of metabolic impairment in cerebral white matter. Magnetic resonance spectroscopy may be useful for evaluation of CNS impairment in patients with OSA. Further investigation is needed to determine the relations between cerebral circulatory and metabolic impairment, and cognitive and neuropsychological dysfunction in patients with OSA.

This study was supported in part by a Special Coordination Fund for Promoting Science and Technology from The Science and Technology Agency of Japan. Dr Kamba was supported by
The Ministry of Education, Science, Sports and Culture of Japan and Konica Corporation.

1 Kales A, Caldwell AB, Cadieux RJ, et al. Severe obstructive seep apnea, II: associated psychopathology and psychosocial consequences. $\mathcal{F}$ Chronic Dis 1985;38:427-34

2 Findley LJ, Barth JT, Powers DC, et al. Cognitive impairment in patients with obstructive sleep apnea and associated hypoxemia. Chest 1986;90:686-90.

3 Greenberg GD, Watson RK, Deptula D. Neuropsychological dysfunction in sleep apnea. Sleep 1987;10:254-62.

4 Montplaisir J, Bédard MA, Richer F, et al. Neurobehavioral manifestations in obstructive sleep apnea syndrome before and after treatment with continuous positive airway pressure. Sleep 1992;15:S17-19.

5 Valencia-Flores M, Bliwise DL, Guilleminault C, et al. Cognitive function in patients with sleep apnea after acute nocturnal nasal continuous positive airway pressure (CPAP) treatment: sleepiness and hypoxemia effects. $\mathcal{F}$ Clin Exp Neuropsychol 1996;18:197-210.

6 Kim HC, Young T, Matthews CG, et al. Sleep-disordered breathing and neuropsychological deficits: a populationbased study. Am f Respir Crit Care Med 1997;156:1813-9.

7 Carskadon MA, Dement WC, Mitler MM, et al. Guidelines for the multiple sleep latency test (MSLT): a standard measure of sleepiness. Sleep 1986;9:519-24.

8 Poceta JS, Timms RM, Jeong DU, et al. Maintenance of wakefulness test in obstructive sleep apnea syndrome. Chest 1992;101:893-7.

9 Walsleben JA, Squires NK, Rothenberger VL. Auditory event-related potentials and brain dysfunction in sleep apnea. Electroencephalogr Clin Neurophysiol 1989;74:297311.

10 Rumbach L, Krieger J, Kurtz D. Auditory event-related potentials in obstructive sleep apnea: effects of treatment with nasal continuous positive airway pressure. Electroencephalogr Clin Neurophysiol 1991;80:454-7.

11 Sangal RB, Sangal JM. P300 latency: abnormal in sleep apnea with somnolence and idiopathic hypersomnia, but normal in narcolepsy. Clin Electroencephalogr 1995;26:14653.

12 Kamba M, Suto Y, Ohta Y, et al. Cerebral metabolism in sleep apnea: evaluation by magnetic resonance spectroscopy. Am 7 Respir Crit Care Med 1997;156:296-8.

13 Larsson HBW, Christiansen P, Jensen M, et al. Localized in vivo proton spectroscopy in the brain of patients with multiple sclerosis. Magn Reson Med 1991;22:23-31.

14 Meyerhoff DJ, Mackay S, Constans J-M, et al. Axonal injury and membrane alterations in Alzheimer's disease suggested and membrane alterations in Alzheimer's disease suggested ing. Ann Neurol 1994;36:40-7.

15 van der Grond J, Balm R, Kappelle LJ, et al. Cerebral metabolism of patients with stenosis or occlusion of the internal carotid artery, a ${ }^{1} \mathrm{H}-\mathrm{MR}$ spectroscopic imaging study. Stroke 1995;26:822-8.

16 Kamba M, Inoue Y, Higami S, et al. Changes in cerebral lactate metabolism in patients with obstructive sleep apnea [abstract]. The Sixth World Congress on Sleep Apnea. Sydney: 2000:68.

17 Young T, Peppard P, Palta M, et al. Population-based study of sleep-disordered breathing as a risk factor for hypertension. Arch Intern Med 1997;157:1746-52.

18 Peker Y, Kraiczi H, Hedner J, et al. An independent association between obstructive sleep apnoea and coronary artery disease. Eur Respir f 1999;14:179-84.

19 Katsumata K, Okada T, Miyao M, et al. High incidence of sleep apnea syndrome in a male diabetic population. sleep apnea syndrome in a male

20 Sappey-Marinier, Angelié E, Meltz P, et al. Brain metabolic changes during normal aging detected by proton $M R$ spectroscopic imaging [abstract]. Proceedings of the Fourth Scientific Meeting and Exhibition of the International Society for Magnetic Resonance in Medicine. Berkeley, CA, USA: The International Society for Magnetic Resonance in Medicine, 1996:926.

21 Guilleminault C, Tilkian A, Dement WC. The sleep apnea syndromes. Anпи Rev Med 1976;27:465-84.

22 Gastaut H, Tassinari CA, Duron B. Etude polygraphique des manifestations episodiques, (hypniques et respiratoires), diurnes et nocturnes, du syndrome de Pickwick. Rev Neurol 1965;112:568-79.

23 The American Academy of Sleep Medicine Task Force. Sleep-related breathing disorders in adults: recommendation for syndrome definition and measurement techniques in clinical research. Sleep 1999;22:667-89.

24 Chimowitz MI, Estes ML, Furlan AJ, et al. Further observations on the pathology of subcortical lesions identified on magnetic resonance imaging. Arch Neurol 1992;49:747-52.

25 Sauter R, Schneider M, Wicklow K, et al. Current status of clinically relevant techniques in magnetic resonance spectroscopy. Electromedica 1992;60:32-54

26 Manabe A. Multi-angle projection shim (MAP shim): in vivo shim adjustment up to 2 nd order with 0.2 second sequence time [abstract]. Proceedings of the Second Annual Meeting of the Society for Magnetic Resonance Berkeley, CA, USA: 1994:65.

27 Birken DL, Oldendorf WH. N-acetyl-L-aspartic acid: a literature review of a compound prominent in ${ }^{1} \mathrm{H}-\mathrm{NMR}$ spectroscopic studies of brain. Neursci Biobehav Rev 1989; 13:23-31.

28 Miller BL. A review of chemical issues in ${ }^{1} \mathrm{H}$ NMR spectroscopy: N-acetyl-L-aspartate, creatine and choline. NMR Biomed 1991;4:47-52. 
29 Fischer AO, Chaudhary BA, Taormina MA, et al. Intracranial hemodynamics in sleep apnea. Chest 1992;102:1402-6. nial hemodynamics in sleep apnea. Chest 1992;102:1402-6.
Bålfors EM, Franklin KA. Impairment of cerebral perfusion Bălfors EM, Franklin KA. Impairment of cerebral perfusion
during obstructive sleep apneas. Am $\mathcal{f}$ Respir Crit Care Med

31 Diomedi M, Placidi F, Cupini LM, et al. Cerebral hemodynamic changes in sleep apnea syndrome and effect of continuous positive airway pressure treatment. Neurology 1998;51:1051-6.

32 Netzer N, Werner P, Jochums I, et al. Blood flow of the middle cerebral artery with sleep-disordered breathing: correlation with obstructive hypopneas. Stroke 1998;29:87-93.

33 Moody DM, Bell MA, Challa VR. Features of the cerebra vascular pattern that predict vulnerability to perfusion or oxygenation deficiency: an anatomic study. Am $\mathcal{f}$ Neuroradiol 1990;11:431-9.

34 Furuta A, Ishii N, Nishihara Y, et al. Medullary arteries in aging and dementia. Stroke 1991;22:442-6.

35 Pantoni L, Garcia JH. Pathogenesis of leukoaraiosis, a review. Stroke 1997;28:652-9.

36 Schmidt R, Fazekas F, Offenbacher H, et al. Neuropsychologic correlates of MRI white matter hyperintensities: a study of 150 normal volunteers. Neurology 1993;43:2490-4.

37 Ylikoski R, Ylikoski A, Erkinjuntti T, et al. White matter changes in healthy elderly persons correlate with attention and speed of mental processing. Arch Neurol 1993;50:818-

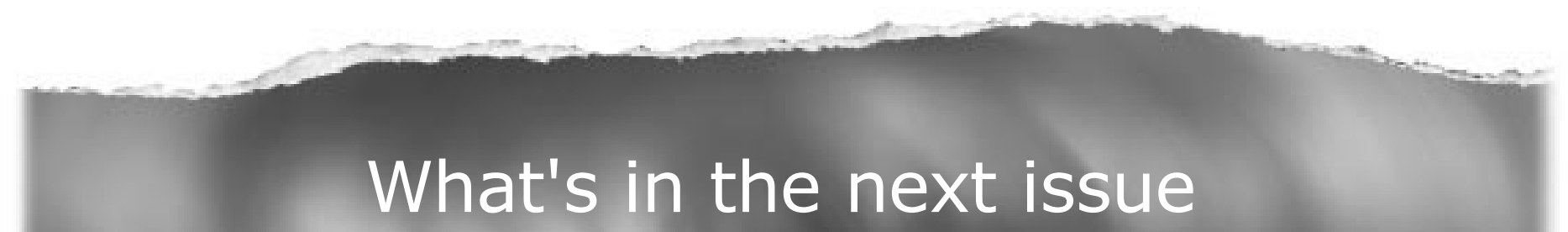

\section{Future content}

See which articles have just been accepted for publication and preview the table of contents for the next issue a month before it is published

www.jnnp.com 